\title{
Short Score Certificates for Upset Tournaments
}

\author{
Jeffrey L. Poet and Bryan L. Shader \\ Department of Mathematics \\ University of Wyoming \\ Laramie, Wyoming 82071, \\ jpoet@uwyo.edu, bshader@uwyo.edu.
}

Submitted: January 15, 1998; Accepted: May 5, 1998.

\begin{abstract}
A score certificate for a tournament, $T$, is a collection of $\operatorname{arcs}$ of $T$ which can be uniquely completed to a tournament with the same scorelist as $T$ 's, and the score certificate number of $T$ is the least number of arcs in a score certificate of $T$. Upper bounds on the score certificate number of upset tournaments are derived. The upset tournaments on $n$ vertices are in one-to-one correspondence with the ordered partitions of $n-3$, and are "almost" transitive tournaments. For each upset tournament on $n$ vertices a general construction of a score certificate with at most $2 n-3$ arcs is given. Also, for the upset tournament, $T_{\lambda}$, corresponding to the ordered partition $\lambda$, a score certificate with at most $n+2 k+3$ arcs is constructed, where $k$ is the number of parts of $\lambda$ of size at least 2 . Lower bounds on the score certificate number of $T_{\lambda}$ in the case that each part is sufficiently large are derived. In particular, the score certificate number of the so-called nearly transitive tournament on $n$ vertices is shown to be $n+3$, for $n \geq 10$.
\end{abstract}

\section{Introduction}

Some recent research has been concerned with the problem of efficiently conveying the information contained in a binary relation $[\mathrm{AR}, \mathrm{KTF}, \mathrm{R}]$. In this paper we continue this line of research by studying the problem for a specific 
class of binary relations. Throughout we use the graph-theoretic notation and terminology in [CL].

Let $V=\left\{v_{1}, v_{2}, \ldots, v_{n}\right\}$. A tournament, $T$, on $V$ is a directed graph obtained by replacing each edge of the complete graph on $V$ with a directed arc. Thus, a tournament represents a complete, asymmetric, irreflexive binary relation. Throughout we use the notation and terminology for tournaments in $[\mathrm{M}]$. We denote the arc from vertex $v_{i}$ to vertex $v_{j}$ by the ordered pair $\left(v_{i}, v_{j}\right)$. The score of a vertex $v_{j} \in V$ is its outdegree. The score-list of $T$ is the multiset of outdegrees of the vertices of $T$.

In this paper we are concerned with efficiently conveying the information contained in a tournament. More precisely, a score certificate for $T$ is a subset, $D$, of arcs such that $T$ is the unique tournament on $V$ that contains each arc in $D$ and whose score-list is the same as $T$ 's. In other words, a score certificate for $T$ is a collection, $D$, of arcs with the property that $T$ can be unambiguously determined by knowing the arcs of $D$ and the score-list of $T$. The score certificate number of $T$ is the least number of arcs in a score certificate for $T$, and is denoted by $\operatorname{sc}(T)$.

Score certificates were first studied in $[\mathrm{FKT}, \mathrm{KTF}]$, where it is proven that if $T$ is not a regular tournament on 3 or 5 vertices, then

$$
n-1 \leq \operatorname{sc}(T) \text {. }
$$

Thus, to convey the results of a tournament on $n$ vertices (via score certificates) at least $n-1$ arcs are required in all but the two exceptional cases. Note that the score certificate number of the transitive tournament on $n$ vertices is $n-1$. In [AR], it is shown that there exists an $\epsilon>0$ independent of $n$ such that

$$
s c(T) \leq(1 / 2-\epsilon) n^{2}
$$

for all tournaments $T$ of order $n$. Also, in $[\mathrm{AR}]$ it is noted that there exist tournaments on $n$ vertices with score certificates at least $(7 / 24+o(1)) n^{2}$. In $[\mathrm{FKT}]$ it is shown that

$$
n-1 \leq \operatorname{sc}\left(N_{n}\right) \leq 3 n-9
$$

where $N_{n}$ is the tournament with vertices $\left\{v_{1}, v_{2}, \ldots, v_{n}\right\}$, and $\operatorname{arcs}\left(v_{1}, v_{n}\right)$ and $\left(v_{i}, v_{j}\right)$ for all other $\left\{v_{i}, v_{j}\right\}$ with $i>j$. The tournament $N_{n}$ is called the nearly transitive tournament on $n$ vertices. Other than these results, little is known about the score certificate number. 
In this paper, we study the score certificate number for a special family of tournaments which generalize the nearly transitive tournament. An upset tournament on $n \geq 4$ vertices is a tournament whose score-list is

$$
\{1,1,2,3, \ldots, n-4, n-3, n-2, n-2\} \text {. }
$$

Properties of upset tournaments have been studied in [BL].

In Section 2, we show that

$$
s c(T) \leq 2 n-3
$$

for each upset tournament $T$ on $n$ vertices. The upset tournaments on $n$ vertices possess special structural properties. In particular, they each have a unique hamiltonian cycle and they are in one-to-one correspondence with the ordered partitions of $n-3$. In Section 2, we also give an upper bound on the score certificate number of an upset tournament which is in terms of the corresponding ordered partition. In most cases, this bound improves that of (3). In Section 3, we derive lower bounds for a special family of upset tournaments. As a consequence we show that the score certificate number of the nearly transitive tournament on $n$ vertices equals $n+3$, for $n \geq 10$.

We conclude this introductory section with necessary preliminaries concerning upset tournaments. Clearly, a transitive tournament is not strongly connected and has no 3-cycles. It follows from results in $[\mathrm{M}]$ that each upset tournament on $n$ vertices has exactly $n-23$-cycles, and this is the least among strongly connected tournaments on $n$ vertices. Thus, one can view the upset tournaments as a class of strongly connected tournaments that are "almost" transitive. Since equality holds in (1) for the transitive tournaments, it is natural to examine the score certificate number of upset tournaments.

It follows from the results in $[\mathrm{BL}]$ that the upset tournaments have a special structure, which we now describe. An ordered partition of the positive integer $\ell$ is a tuple of positive integers whose sum is $\ell$. An ordered partition $\lambda=\left(p_{1}, p_{2}, \ldots, p_{k}\right)$ of $n-3$ determines a tournament $T_{\lambda}$ on $n$ vertices as follows. Let $V=\left\{v_{1}, \ldots, v_{n}\right\}$ and let $v_{i}$ and $v_{j}$ be vertices with $i<j$. If $\left(v_{i}, v_{j}\right)$ is $\left(v_{1}, v_{2}\right),\left(v_{n-1}, v_{n}\right)$ or one of

$$
\left(v_{2}, v_{2+p_{1}}\right),\left(v_{2+p_{1}}, v_{2+p_{1}+p_{2}}\right), \ldots,\left(v_{2+p_{1}+\cdots+p_{k-1}}, v_{2+p_{1}+\cdots+p_{k-1}+p_{k}}\right),
$$

then $\left(v_{i}, v_{j}\right)$ is an arc of $T_{\lambda}$. Otherwise, $\left(v_{j}, v_{i}\right)$ is an arc of $T_{\lambda}$. It is easy to verify that $T_{\lambda}$ is an upset tournament. For example, the nearly transitive 
tournament on $n$ vertices is isomorphic to $T_{(n-3)}$. It follows from results in $[\mathrm{BL}]$ that the nonisomorphic upset tournaments of order $n$ are precisely the $T_{\lambda}$ where $\lambda$ runs over all ordered partitions of $n-3$. Hence, there are exactly $2^{n-4}$ non-isomorphic upset tournaments of order $n$.

An $\operatorname{arc}\left(v_{i}, v_{j}\right)$ of a tournament $T$ is an upset arc provided the score of $v_{i}$ is less than or equal to that of $v_{j}$. The upset arcs of $T_{\lambda}$ are precisely the arcs $\left(v_{1}, v_{2}\right),\left(v_{n-1}, v_{n}\right)$, and $\left(v_{2+p_{1}+p_{2}+\cdots+p_{j}}, v_{2+p_{1}+p_{2}+\cdots+p_{j}+p_{j+1}}\right)$ for $j=$ $0,1,2, \ldots, k-1$. Note that upset arcs of an upset tournament form a path, which we call its upset path.

An upset tournament $T$ with vertices $v_{1}, \ldots, v_{n}$ is in standard form provided $\left(v_{i}, v_{j}\right)$ with $i<j$ is an arc of $T$ if and only if $\left(v_{i}, v_{j}\right)$ is in $T$ 's upset path. Thus, each $T_{\lambda}$ is in standard form, and each upset tournament can be put into standard form by relabelling its vertices.

Pictorially, each upset tournament, $T$, in standard form can be constructed by placing the vertices $v_{1}, v_{2}, \ldots, v_{n}$ in order from bottom to top on a vertical line, drawing an path from $v_{1}$ to $v_{n}$ that goes through $v_{2}$ and $v_{n-1}$ and consists entirely of upward-oriented arcs, and orienting all other arcs downward. Moreover, up to isomorphism, the upset tournaments are precisely the tournaments which have this type of picture. For example, the upset tournaments with 6 vertices (in standard form) are given in Figure 1. Here, as is customary when illustrating tournaments, only the upwardoriented arcs are shown.
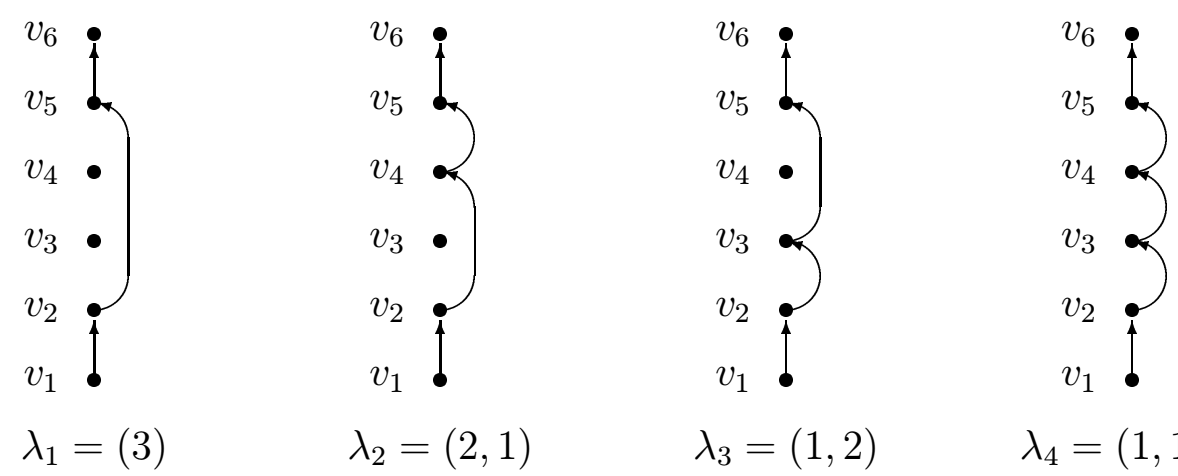

$\lambda_{1}=(3)$

$$
\lambda_{2}=(2,1)
$$

$$
\lambda_{3}=(1,2)
$$$$
\lambda_{4}=(1,1,1)
$$

Figure 1 
THE ELECTRONIC JOURNAL OF COMBINATORICS 5 (1998), \#R24

\section{Upper bounds}

In this section we derive two linear upper bounds on the score certificate number of an arbitrary upset tournament on $n$ vertices. The first does not depend upon the partition corresponding to the tournament, but the second does. We begin by describing certain arcs that are contained in each score certificate of an upset tournament.

Let $T$ be an upset tournament of order $n$. We define a forced arc of $T$ to be an $\operatorname{arc}\left(v_{i}, v_{j}\right)$ of $T$ where the score of $v_{i}$ is 1 more than the score of $v_{j}$. If $\left(v_{i}, v_{j}\right)$ is a forced arc, then the tournament obtained from $T$ by reversing this arc is also an upset tournament. Thus, every score certificate of $T$ contains each forced arc of $T$.

It is easy to determine the forced arcs of the upset tournament $T_{\lambda}$ corresponding to the ordered partition $\lambda=\left(p_{1}, p_{2}, \ldots, p_{k}\right)$ of $n-3$. When $n=4$, all non-upset arcs are forced arcs. Otherwise the forced arcs of $T_{\lambda}$ are $\left(v_{n}, v_{n-2}\right),\left(v_{3}, v_{1}\right)$, and each of the arcs in each of the paths

$$
v_{2+p_{1}+\cdots+p_{j-1}+p_{j}}, v_{2+p_{1}+\cdots+p_{j-1}+p_{j}-1}, v_{2+p_{1}+\cdots+p_{j-1}+p_{j}-2}, \ldots, v_{2+p_{1}+\cdots+p_{j-1}}
$$

where $p_{j} \geq 2$. For example, the forced arcs of the nearly transitive tournament on $n \geq 5$ vertices are the $\operatorname{arcs}\left(v_{n}, v_{n-2}\right),\left(v_{3}, v_{1}\right)$, and the arcs in the path $v_{n-1}, v_{n-2}, \ldots, v_{2}$.

We call the two vertices of an upset tournament with outdegree 1 the bottom vertices and the two vertices with indegree 1 the top vertices. The following shows that each upset tournament can be unambiguously determined given its bottom vertices and any collection of its arcs which contains each upset arc and each forced arc.

Lemma 2.1 Let $T$ be an upset tournament in standard form with vertices $v_{1}, v_{2}, \ldots, v_{n}$. Let $\alpha$ be a subset of arcs of $T$ containing each forced arc and each upset arc. Let $T^{\prime}$ be an upset tournament with vertices $v_{1}, v_{2}, \ldots, v_{n}$ such that $v_{1}$ and $v_{2}$ are the bottom vertices of $T^{\prime}$ and each arc in $\alpha$ is an arc of $T^{\prime}$. Then $T^{\prime}=T$.

Proof. Let $D$ be the digraph with vertices $v_{1}, v_{2}, \ldots, v_{n}$ whose arcs are those in $\alpha$, and let $\gamma$ be the set of arcs in $T$ that are not in $T^{\prime}$. Note that $T^{\prime}$ is the tournament obtained from $T$ by reversing the $\operatorname{arcs}$ in $\gamma$. 
We claim that $\gamma$ is empty, and hence $T^{\prime}=T$. For suppose not. Since either $\left(v_{j}, v_{j+1}\right)$ or $\left(v_{j+1}, v_{j}\right)$ is in $\alpha$ for $j=1, \ldots, n-1$ and $\alpha$ contains $T$ 's upset arcs, all arcs in $\gamma$ have the form $\left(v_{j}, v_{i}\right)$ where $j-1>i$. Among all the arcs in $\gamma$ choose one, say $\left(v_{j}, v_{i}\right)$, such that $i$ is minimum. Since $v_{1}$ and $v_{2}$ are the bottom vertices of $T$ and of $T^{\prime}, i \notin\{1,2\}$. Since neither $v_{n-1}$ nor $v_{n}$ is a terminal vertex of an arc in $\gamma, i \leq n-2$. Thus, the score of $v_{i}$ in $T$ is $i-1$.

We claim that no vertex, $v_{k}$, in $T^{\prime}$ has score equal to $i-1$. If $k<i$, then the choice of $\left(v_{j}, v_{i}\right)$ implies that the scores of $v_{k}$ in $T$ and in $T^{\prime}$ are equal, and hence less than $i-1$. If $k=i$, then the score of $v_{i}$ in $T$ is less than its score in $T^{\prime}$. Suppose that $i<k<n$. Then the score of $v_{k}$ in $T$ is $k-1$. There are at most $k-i-1$ arcs in $\gamma$ of the form $\left(v_{k}, v_{\ell}\right)$ where $k-1>\ell \geq i$. Thus, the score of $v_{k}$ in $T^{\prime}$ is at least $k-1-(k-i-1)=i$. Finally, suppose that $k=n$. Then the score of $v_{k}$ in $T$ is $n-2$. The arcs $\left(v_{n}, v_{n-1}\right)$, and $\left(v_{n}, v_{n-2}\right)$ are not in $\gamma$. It follows that the score of $v_{n}$ in $T^{\prime}$ is at least $n-2-(n-i-2)=i$. Therefore, we have reached the contradiction that no vertex in $T^{\prime}$ has score $i-1$.

We conclude that $\gamma$ is empty, and hence that $T$ is the unique upset tournament which contains $\alpha$ and has bottom vertices $v_{1}$ and $v_{2}$.

We now use Lemma 2.1 to construct a score certificate with $2 n-3$ arcs for each upset tournament on $n$ vertices.

Theorem 2.2 Let $T$ be an upset tournament on $n$ vertices. Then

$$
s c(T) \leq 2 n-3 .
$$

Proof. Without loss of generality we may assume that $T$ is in standard form. It is easy to verify the result for $T=T_{(1)}, T=T_{(1,1)}$ and $T=T_{(2)}$. Hence, we may assume that $n \geq 6$.

Let $\alpha$ be the set consisting of the upset arcs and the forced $\operatorname{arcs}$ of $T$. Consider the digraph, $D$, whose vertices are those of $T$ and whose arcs are those in $\alpha$. We first determine the outdegree of each vertex in $D$.

The vertices $v_{1}, v_{2}$ and $v_{n}$ each have outdegree 1 in $D$. The outdegree of $v_{3}$ in $D$ is 2 . If $v_{j}$ is a vertex which is not on the upset path and $4 \leq j<n$, then $\left(v_{j}, v_{j-1}\right)$ is a forced arc of $T$ and $v_{j}$ has outdegree 1 in $D$. Otherwise, $v_{j}$ is a vertex on the upset path with $4 \leq j<n$. Let $\left(v_{i}, v_{j}\right)$ be the upset arc 
whose terminal vertex is $v_{j}$. If $j-i=1$, then the outdegree of $v_{j}$ in $D$ is 1 . If $j-i \neq 1$, then $\left(v_{j}, v_{j-1}\right)$ is a forced arc of $T$, and the outdegree of $v_{j}$ in $D$ is 2 .

Since $n \geq 6$, it can be verified that at least one of $v_{4}$ or $v_{5}$ has outdegree 1 in $D$. Let $m$ be the smallest index such that $m \notin\{1,2, n\}$ and $v_{m}$ has outdegree 1 in $D$. Note that $m \geq 4$. Let

$$
\beta=\left\{\left(v_{j}, v_{j-2}\right): m<j<n \text {, and } v_{j} \text { has outdegree } 1 \text { in } D\right\} \cup\left\{\left(v_{n}, v_{n-3}\right)\right\} .
$$

It is easy to verify that $\beta$ is a collection of $\operatorname{arcs}$ in $T$ that are not in $\alpha$. Let $D^{\prime}$ be the digraph whose vertices are $v_{1}, v_{2}, \ldots, v_{n}$ and whose arcs are those in $\alpha \cup \beta$. By construction, each vertex in $D^{\prime}$ other than $v_{1}, v_{2}, v_{m}$ has outdegree 2 , and each of these 3 vertices has outdegree 1 . Hence $\alpha \cup \beta$ has $2 n-3$ arcs.

We show that $\alpha \cup \beta$ is a score certificate for $T$. Suppose that $T^{\prime}$ is an upset tournament with vertices $v_{1}, v_{2}, \ldots, v_{n}$ which has all the arcs in $\alpha \cup \beta$. We show that $T^{\prime}$ is $T$.

The vertices of outdegree 1 in $D^{\prime}$ are $v_{1}, v_{2}$, and $v_{m}$. Also, $\left(v_{1}, v_{2}\right)$ is an arc of $D^{\prime}$, the arc in $D^{\prime}$ with initial vertex $v_{2}$ does not terminate at $v_{m}$, and the arc in $D^{\prime}$ with initial vertex at $v_{m}$ does not terminate at $v_{1}$ or $v_{2}$. If the outdegree of $v_{m}$ in $T^{\prime}$ is 1 , then $\left(v_{1}, v_{m}\right)$ and $\left(v_{2}, v_{m}\right)$ are $\operatorname{arcs}$ of $T^{\prime}$, and we are led to the contradiction that $v_{m}$ is the only vertex in $T^{\prime}$ with outdegree 1. Hence $v_{1}$ and $v_{2}$ are the bottom vertices of $T^{\prime}$. Lemma 2.1 now implies that $T^{\prime}=T$, and thus $\alpha \cup \beta$ is a score certificate for $T$.

Later we will show that the bound in Theorem 2.2 is not sharp in general. The construction of score certificates for upset tournaments given in the proof of Theorem 2.2 makes little use of the special structure of upset tournaments. We now present a construction which exploits the following property.

Proposition 2.3 Let $T$ be an upset tournament on $n$ vertices. Then $T$ contains a unique hamiltonian cycle.

Proof. Without loss of generality we may assume that the vertices of $T$ are labelled so that $T$ is in standard form. Since $T$ is strongly connected, it is well-known (see $[\mathrm{M}]$ ) that $T$ has a hamiltonian cycle.

Let $H$ denote a hamiltonian cycle in $T$. The upset path must be a subpath of $H$, since it is the unique path from $v_{1}$ to $v_{n}$. All other $\operatorname{arcs}\left(v_{i}, v_{j}\right)$ in $T$ have 
$i>j$. Thus, the subpath of $H$ from $v_{n}$ to $v_{1}$ must be the path which starts at $v_{n}$, traverses through the vertices not on the upset path in decreasing order, and ends at $v_{1}$. Therefore, $H$ is unique.

Let $T$ be a tournament with a hamiltonian cycle $H$. For vertices $u$ and $v$, we denote the subpath of $H$ from $u$ to $v$ by $u H v$. If $\left(v_{i}, v_{j}\right)$ and $\left(v_{k}, v_{\ell}\right)$ are arcs of $T$ not in $H$, we say that $\left(v_{k}, v_{\ell}\right)$ is nested inside $\left(v_{i}, v_{j}\right)$ provided $v_{i} H v_{j}$ contains $v_{k} H v_{\ell}$. The following lemma is useful in identifying the location of the bottom vertices along the hamiltonian cycle of an upset tournament.

Lemma 2.4 Let $T$ be an upset tournament with hamiltonian cycle $H$. Suppose $\left(v_{i}, v_{j}\right)$ and $\left(v_{k}, v_{\ell}\right)$ are arcs of $T$ not in $H$ such that $\left(v_{k}, v_{\ell}\right)$ is nested inside $\left(v_{i}, v_{j}\right)$. Then neither of the bottom vertices of $T$ is on $v_{i} H v_{k}$.

Proof. Neither $v_{i}$ nor $v_{k}$ is a bottom vertex, since each is the initial vertex of an arc in $H$ and an arc not in $H$. Similarly, neither $v_{j}$ nor $v_{\ell}$ is a top vertex.

Suppose to the contrary that a bottom vertex $w$ is on $v_{i} H v_{k}$. Then T's top vertices must occur on $w H v_{\ell}$, for otherwise, $\left(v_{k}, v_{\ell}\right)$ is an upset arc not in $H$. Hence, the scores of the vertices along $v_{\ell} H v_{i}$ are in decreasing order. In particular, the score of $v_{j}$ is greater than that of $v_{i}$, which leads to the contradiction that $\left(v_{i}, v_{j}\right)$ is an upset arc of $T$ not in $H$.

We now note that each of the six arcs in the upset tournament $T_{(1)}$ is a forced arc or is on the hamiltonian cycle. Hence, $T_{(1)}$ has a score certificate consisting of the forced arcs and the arcs in its hamiltonian cycle. For upset tournaments on five or more vertices we have the following result.

Theorem 2.5 Let $T$ be an upset tournament on $n \geq 5$ vertices. There exists a score certificate for $T$ consisting of the arcs in its unique hamiltonian cycle, its forced arcs, and one other arc.

Proof. Let $H$ be the hamiltonian cycle of $T$. Without loss of generality we may assume $T=T_{\lambda}$ where $\lambda=\left(p_{1}, p_{2}, \ldots, p_{k}\right)$. Let $\beta$ be the set consisting of the arcs in $H$ and the forced arcs of $T$.

By Lemma 2.1, it suffices to show that there exists an arc $e$ such that each upset tournament, $T^{\prime}$, on the same vertices as $T$ which contains $e$ and the arcs in $\beta$ has the same bottom vertices as $T$. 
THE ELECTRONIC JOURNAL OF COMBINATORICS 5 (1998), \#R24

The choice of the arc $e$ depends upon $\lambda$. We consider four cases.

CASE 1: Each $p_{i}$ equals 1.

Since $n \geq 5$, the arc $\left(v_{n-1}, v_{1}\right)$ is in $T$ but not in $\beta$. Take $e$ to be this arc. Note that $e$ is nested inside the $\operatorname{arc}\left(v_{3}, v_{1}\right)$ of $T$, and that $\left(v_{3}, v_{1}\right)$ is not in $H$. Applying Lemma 2.4, we conclude that none of the vertices $v_{3}, v_{4}, \ldots, v_{n-1}$ is a bottom vertex of $T^{\prime}$. Since $v_{n}$ is the initial vertex of two arcs in $\beta, v_{n}$ is not a bottom vertex of $T^{\prime}$. Hence, $T^{\prime}$ has the same bottom vertices as $T$.

CASE 2: $\quad$ For some $j \geq 2, p_{1}=p_{2}=\cdots=p_{j-1}=1$ and $p_{j} \geq 2$.

Then $\left(v_{j+2}, v_{j+1}\right)$ is a forced arc of $T,\left(v_{j+2}, v_{1}\right) \in H$ and there exists a unique vertex $v_{q}$ such that $\left(v_{q}, v_{j+2}\right) \in H$. Clearly, $\left(v_{q}, v_{1}\right)$ is an arc of $T$ not in $H$. Take $e$ to be this arc. Note that $e$ is nested inside the $\operatorname{arc}\left(v_{3}, v_{1}\right)$, and that $\left(v_{3}, v_{1}\right)$ is not in $H$. Applying Lemma 2.4, we conclude that the bottom vertices of $T^{\prime}$ are contained in $\left\{v_{1}, v_{2}, v_{j+2}\right\}$. Since $\left(v_{j+2}, v_{1}\right)$ and $\left(v_{j+2}, v_{j+1}\right)$ are in $\beta, v_{j+2}$ is not a bottom vertex of $T^{\prime}$. Hence, $T^{\prime}$ has the same bottom vertices as $T$.

CASE 3: $\quad k=1$.

Then $p_{1}=n-3$, and since $n \geq 5$ the $\operatorname{arc}\left(v_{n}, v_{2}\right)$ is in $T$ and not in $\beta$. Take $e$ to be this arc. Note that $\left(v_{3}, v_{2}\right)$ is an arc of $\beta$ not in $H$, and is nested inside $e$. By Lemma 2.4, we conclude that the bottom vertices of $T^{\prime}$ are contained in $\left\{v_{1}, v_{2}, v_{n-1}\right\}$. Since $\left(v_{n-1}, v_{n}\right)$ and $\left(v_{n-1}, v_{n-2}\right)$ are arcs in $\beta, v_{n-1}$ is not a bottom vertex of $T^{\prime}$. Hence, $T^{\prime}$ has the same bottom vertices as $T$.

CASE $4: \quad k \geq 2$ and $p_{1} \geq 2$.

Then $\left(v_{3}, v_{2}\right)$ is a forced $\operatorname{arc}$ of $T$ which is not in $H$. The $\operatorname{arc}\left(v_{2+p_{1}+p_{2}}, v_{2}\right)$ is an arc of $T$ not in $\beta$. Take $e$ to be this arc. Then $\left(v_{3}, v_{2}\right)$ is nested inside $e$. By Lemma 2.4, we conclude that the bottom vertices of $T^{\prime}$ are contained in $\left\{v_{1}, v_{2}, v_{2+p_{1}}\right\}$. Since $\left(v_{2+p_{1}}, v_{2+p_{1}+p_{2}}\right)$ and $\left(v_{2+p_{1}}, v_{1+p_{1}}\right)$ are in $\beta, v_{2+p_{1}}$ is not a bottom vertex of $T^{\prime}$. Hence, $T^{\prime}$ has the same bottom vertices as $T$. 
The desired conclusion is reached in each case, and the proof is complete.

Examples of score certificates, constructed as in the proof of Theorem 2.5, for three upset tournaments on 11 vertices are given in Figure 2. Using Theorem 2.5 we can derive an upper bound on the score certificate number of $T_{\lambda}$.

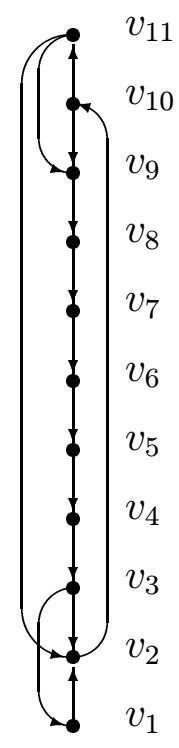

$\lambda=(8)$

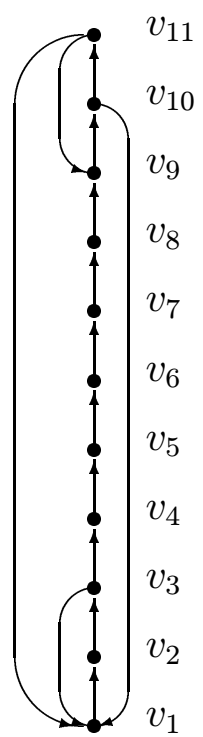

$\lambda=(1,1,1,1,1,1,1,1)$

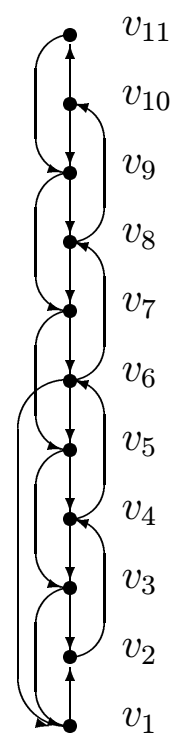

$\lambda=(2,2,2,2)$

Figure 2

Corollary 2.6 Let $\lambda=\left(p_{1}, p_{2}, \ldots, p_{k}\right)$ be an ordered partition of $n-3$, where $n \geq 5$. Then

$$
s c\left(T_{\lambda}\right) \leq n+2 x+y+1
$$

where $x$ equals $\mid\left\{i: 1 \leq i \leq k\right.$ and $\left.p_{i} \geq 2\right\} \mid$, and $y$ equals $\mid\{i: i \in$ $\{1, k\}$ and $\left.p_{i}=1\right\} \mid$.

Proof. By Theorem 2.5, there is a score certificate for $T_{\lambda}$ consisting of the arcs in its hamiltonian cycle, its forced arcs, and one additional arc. The hamiltonian cycle has $n$ arcs, the number of forced arcs not in the hamiltonian 
cycle and not incident to $v_{1}$ or $v_{n}$ is $2 x$, and the number of forced arcs incident to $v_{1}$ or $v_{n}$ and not in the hamiltonian cycle is $y$. The result now follows.

If $\lambda=(2,2, \ldots, 2)$, then by Corollary $2.6, s c\left(T_{\lambda}\right) \leq 2 n-3+1$, and this bound is worse than that given in Theorem 2.2. However, it can be verified that the score certificate for $T_{(2,2, \ldots, 2)}$ constructed in the proof of Corollary 2.6 is not minimal (with respect to set inclusion). For example, one can delete the $\operatorname{arcs}\left(v_{6}, v_{1}\right)$ and $\left(v_{10}, v_{11}\right)$ from the score certificate for $T_{(2,2,2,2)}$ shown in Figure 2 and still have a score certificate.

For arbitrary $\lambda$, it is easy to see that the bound for $\operatorname{sc}\left(T_{\lambda}\right)$ in Corollary 2.6 is usually better than that in Theorem 2.2. Indeed, Corollary 2.6 implies that the score certificate number of the nearly transitive tournament on $n$ vertices is at most $n+3$.

\section{Lower bounds}

In this section we derive lower bounds for the score certificate number of certain upset tournaments. In particular, we show that the score certificate number of the nearly transitive tournament on $n$ vertices equals $n+3$, for $n \geq 10$.

The class of upset tournaments that we study in this section is the class of $T_{\lambda}$ where each part $p_{i}$ of $\lambda=\left(p_{1}, \ldots, p_{k}\right)$ is at least $k+6$. For such tournaments, Corollary 2.6 guarantees a score certificate with $n+2 k+1$ arcs. We begin by showing that for such a tournament, each score certificate with less than $n+2 k+1$ arcs contains each arc in its upset path, other than $\left(v_{1}, v_{2}\right)$ and $\left(v_{n-1}, v_{n}\right)$. For convenience, we define the truncated upset path of $T_{\lambda}$ to be the path obtained from its upset path by removing the first and last arc.

Lemma 3.1 Let $\lambda=\left(p_{1}, \ldots, p_{k}\right)$ be an ordered partition of $n-3$ such that each $p_{i} \geq k+6$. Then each score certificate for $T_{\lambda}$ of cardinality less than $n+2 k+1$ contains each arc in $T_{\lambda}$ 's truncated upset path.

Proof. Suppose to the contrary that there exists a score certificate, $D$, for $T_{\lambda}$ with less than $n+2 k+1$ arcs that does not contain each arc in the truncated upset path. Note that if $\gamma$ is a cycle of $T_{\lambda}$, then the tournament 
obtained from $T_{\lambda}$ by reversing the arcs of $\gamma$ has the same score-list as $T_{\lambda}$. Since $D$ is a score certificate, it follows that $D$ contains at least one arc in each cycle of $T_{\lambda}$.

For $m=1,2, \ldots, k$, let $x_{m}$ be the number of non-forced arcs in $D$ having both initial and terminal vertices in

$$
\left\{v_{r}, v_{r+1}, \ldots, v_{t}\right\}
$$

where $r=2+p_{1}+p_{2}+\cdots+p_{m-1}$ and $t=2+p_{1}+p_{2}+\cdots+p_{m}$. First suppose that the upset $\operatorname{arc}\left(v_{r}, v_{t}\right)$ is not in $D$. For each $s$ with $r+1<s<t-1$,

$$
v_{t} \rightarrow v_{s} \rightarrow v_{r} \rightarrow v_{t}
$$

is a 3 -cycle in $T_{\lambda}$, and hence $D$ contains at least one of the non-forced arcs $\left(v_{t}, v_{s}\right)$ and $\left(v_{s}, v_{r}\right)$. Therefore, $x_{m} \geq p_{m}-3 \geq k+3$. If $\left(v_{r}, v_{t}\right)$ is in $D$, then certainly $x_{m} \geq 1$.

It follows that the number of non-forced $\operatorname{arcs}$ in $D$ is at least

$$
\sum_{m=1}^{k} x_{m} \geq(k-1)+k+3=2 k+2
$$

Since $D$ contains the $n-1$ forced arcs, we are led to the contradiction that $D$ has at least $n+2 k+1$ arcs.

Let $\lambda=\left(p_{1}, \ldots, p_{k}\right)$ be an ordered partition of $n-3$ with each $p_{i} \geq k+6$. An immediate consequence of Lemma 3.1 is that

$$
s c\left(T_{\lambda}\right) \geq n+k-1 .
$$

In the remainder of this paper we show that this bound can be improved to

$$
s c\left(T_{\lambda}\right) \geq n+k+2 .
$$

The technique employed is as follows. Let $\delta$ be the set consisting of the arcs in the truncated upset path of $T_{\lambda}$ and the forced arcs of $T_{\lambda}$. We exhibit a family $T_{1}, \ldots, T_{5}$ of upset tournaments on the vertices $v_{1}, \ldots, v_{n}$ with the following properties:

(a) $T_{i} \neq T_{\lambda}$ for $i=1,2,3,4,5$, and 
(b) for each pair $e$ and $f$ of $\operatorname{arcs}$ which are in $T_{\lambda}$ and not in $\delta$, there exists a $j$ such that both $e$ and $f$ are $\operatorname{arcs}$ of $T_{j}$.

We claim that this implies that $s c\left(T_{\lambda}\right) \geq n+k+2$. To see this, let $D$ be a score certificate for $T_{\lambda}$. If $D$ does not contain the truncated upset path of $T_{\lambda}$, then Lemma 3.1 implies that $D$ has at least $n+2 k+1$ arcs. Otherwise, $D$ contains each arc in $\delta$. The existence of such $T_{1}, \ldots, T_{5}$ implies that no set of arcs consisting of $\delta$ and at most two other arcs is a score certificate for $T_{\lambda}$. Thus, $D$ has at least $3 \operatorname{arcs}$ not in $\delta$. Therefore, $D$ has at least $n+k+2$ arcs.

In order to easily describe the tournaments $T_{1}, \ldots, T_{5}$, we introduce some notation. Throughout the remainder of the paper we identify the vertex $v_{i}$ with the index $i$. Let $T$ be an upset tournament with vertices $1,2, \ldots, n$ not necessarily in standard form. Let $\pi$ be the permutation so that when $T$ is put in standard form, the vertices from bottom to top are $\pi(1), \pi(2), \ldots, \pi(n)$. If we start with the $n$-tuple $(\pi(1), \pi(2), \ldots, \pi(n))$ and boldface each entry in the $n$-tuple corresponding to a vertex on $T$ 's upset path, we obtain a $n$-tuple which completely describes $T$. For example, the nearly transitive tournament on $n$ vertices in standard form is denoted by the $n$-tuple

$$
(\mathbf{1}, \mathbf{2}, 3,4, \ldots, n-3, n-2, \mathbf{n}-\mathbf{1}, \mathbf{n}),
$$

and the 7 -tuple $(\mathbf{3}, \mathbf{4}, \mathbf{1}, \mathbf{7}, \mathbf{2}, \mathbf{5}, \mathbf{6})$ determines the upset tournament illustrated in Figure 3.

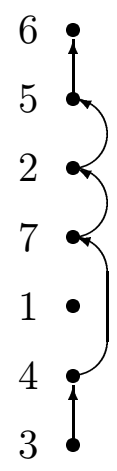

Figure 3 
If $T$ is the tournament described by a given $n$-tuple, then $(i, j)$ is an arc of $T$ if and only if

(1) both $i$ and $j$ are boldfaced and $j$ is the first boldfaced entry to the right of $i$,

(2) both $i$ and $j$ are boldfaced, $j$ is to the left of $i$ and there is at least one boldfaced entry between $j$ and $i$, or

(3) $i$ or $j$ is not boldfaced and $j$ occurs to the left of $i$.

Theorem 3.2 Let $\lambda=\left(p_{1}, \ldots, p_{k}\right)$ be an ordered partition of $n-3$ such that each $p_{i} \geq k+6$. Then $s c\left(T_{\lambda}\right) \geq n+k+2$.

Proof. It suffices to exhibit tournaments $T_{1}, \ldots, T_{5}$ satisfying (a) and (b) above.

Without loss of generality we may assume that $T_{\lambda}$ has upset path 1,2 , $i_{1}, i_{2}, \ldots, i_{k-1}, n-1, n$ and hence is determined by

$$
\begin{aligned}
& \left(\mathbf{1}, \mathbf{2}, 3, \ldots, i_{1}-1,\right. \\
& \mathbf{i}_{\mathbf{1}}, i_{1}+1, \ldots, i_{2}-1, \\
& \mathbf{i}_{\mathbf{2}}, i_{2}+1, \ldots, i_{3}-1, \\
& \quad \vdots \\
& \mathbf{i}_{\mathbf{k}-\mathbf{1}}, i_{k-1}+1, \ldots, n-2, \\
& \mathbf{n}-\mathbf{1}, \mathbf{n}) .
\end{aligned}
$$

Let $T_{1}$ be the upset tournament determined by

$$
\begin{aligned}
& \left(\mathbf{1}, \mathbf{4}, \mathbf{3}, \mathbf{2}, 5, \ldots, i_{1}-1,\right. \\
& \mathbf{i}_{\mathbf{1}}, i_{1}+1, \ldots, i_{2}-1, \\
& \mathbf{i}_{\mathbf{2}}, i_{2}+1, \ldots, i_{3}-1, \\
& \vdots \\
& \mathbf{i}_{\mathbf{k}-\mathbf{1}}, i_{k-1}+1, \ldots, n-2, \\
& \mathbf{n}-\mathbf{1}, \mathbf{n}) .
\end{aligned}
$$

It is easy to verify that $T_{1}$ contains $T_{\lambda}$ 's truncated upset path and forced arcs. Also, the set of arcs in $T_{\lambda}$ that are not in $T_{1}$ is

$$
X=\{(4,2),(4,1)(1,2)\} .
$$


Analogously, we define $T_{2}$ to be the upset tournament determined by

$$
\begin{array}{r}
(\mathbf{1}, \mathbf{2}, \\
3, \ldots, i_{1}-1, \mathbf{i}_{\mathbf{1}}, \\
i_{1}+1, \ldots, i_{2}-1, \mathbf{i}_{\mathbf{2}} \\
\vdots \\
\left.i_{k-1}+1, \ldots, n-4, \mathbf{n}-\mathbf{1}, \mathbf{n}-\mathbf{2}, \mathbf{n}-\mathbf{3}, \mathbf{n}\right) .
\end{array}
$$

and observe that the set of $\operatorname{arcs}$ in $T_{\lambda}$ that are not in $T_{2}$ is

$$
Y=\{(n, n-3),(n-1, n),(n-1, n-3)\} .
$$

Note that $X$ and $Y$ are disjoint. Let $e$ and $f$ be $\operatorname{arcs}$ of $T_{\lambda}$ that are not forced arcs and not in its truncated upset path. If it is not the case that $X$ contains one of $e$ or $f$ and $Y$ contains the other, then the arcs $e$ and $f$ are simultaneously in at least one of $T_{1}$ and $T_{2}$. Thus, we assume without loss of generality that $e \in X$ and $f \in Y$.

Let $T_{3}$ be the upset tournament determined by

$$
\begin{aligned}
& \left(\mathbf{i}_{\mathbf{1}}-\mathbf{1}, \mathbf{i}_{\mathbf{1}}-\mathbf{2}, 1, \mathbf{i}_{\mathbf{1}}-\mathbf{3}, \mathbf{i}_{\mathbf{1}}-\mathbf{4}, \ldots, \mathbf{3}, \mathbf{2},\right. \\
& \mathbf{i}_{\mathbf{1}}, i_{1}+1, i_{1}+2, \ldots, i_{2}-1 \\
& \mathbf{i}_{\mathbf{2}}, i_{2}+1, \ldots, i_{3}-1 \\
& \quad \vdots \\
& \mathbf{i}_{\mathbf{k}-\mathbf{1}}, i_{k-1}+1, \ldots, n-2 \\
& \mathbf{n}-\mathbf{1}, \mathbf{n})
\end{aligned}
$$

where we identify $i_{1}$ and $n-1$ in the case $k=1$. It can be verified that $T_{3}$ contains $T_{\lambda}$ 's truncated upset path and forced arcs. Also $T_{3}$ contains the arcs in $\{(4,1)\} \cup Y$, and hence if $e=(4,1)$ then $T_{3}$ contains both $e$ and $f$.

Analogously, we define $T_{4}$ to be the upset tournament determined by

$$
\begin{array}{r}
(\mathbf{1}, \mathbf{2} \\
3,4, \ldots, i_{1}-1, \mathbf{i}_{\mathbf{1}} \\
3, i_{2}-1, \mathbf{i}_{\mathbf{2}} \\
\vdots \\
i_{1}+1, i_{1}+2, \ldots, i_{k-1}-1, \mathbf{i}_{\mathbf{k}-\mathbf{1}} \\
\left.i_{k-2}+1, i_{k-2}+2, \ldots, \mathbf{i}_{\mathbf{k}-\mathbf{1}}+\mathbf{3}, n, \mathbf{i}_{\mathbf{k}-\mathbf{1}}+\mathbf{2}, \mathbf{i}_{\mathbf{k}-\mathbf{1}}+\mathbf{1}\right)
\end{array}
$$


where we identify vertices 2 and $i_{k-1}$ in the case that $k=1$, and conclude that if $f=(n, n-3)$ then $T_{4}$ contains both $e$ and $f$.

Finally, let $T_{5}$ be the upset tournament determined by

$$
(\mathbf{n}, \mathbf{n}-\mathbf{2}, \mathbf{n}-\mathbf{3}, n-1, \mathbf{n}-\mathbf{4}, \mathbf{n}-\mathbf{5}, \ldots, \mathbf{5}, 2, \mathbf{4}, \mathbf{3}, \mathbf{1}),
$$

where each of the entries between $n-5$ and 5 is boldfaced. It can be verified that $T_{1}$ contains $T_{\lambda}$ 's truncated upset path and forced arcs. Also, $T_{5}$ contains the arcs in $\{(1,2),(4,2),(n-1, n),(n, n-2)\}$. Hence, if $e \neq(4,1)$ and $f \neq(n, n-3)$, then $T_{5}$ contains both $e$ and $f$.

Therefore, the upset tournaments $T_{1}, \ldots, T_{5}$ satisfy property (b), and it is clear that they also satisfy property (a).

In Figure 4, we illustrate the tournaments $T_{1}, \ldots, T_{5}$ which are constructed in the proof of Theorem 3.2 for the upset tournament $T_{(8,8)}$.

The following is an immediate consequence of Corollary 2.6 and Theorem 3.2 .

Corollary 3.3 Let $n \geq 10$, and let $N_{n}$ be the nearly transitive tournament on $n$ vertices. Then

$$
\operatorname{sc}\left(N_{n}\right)=n+3
$$

The following chart summarizes the results for tournaments on $n$ vertices corresponding to the partition $\lambda=\left(p_{1}, \ldots, p_{k}\right)$, where each $p_{i} \geq k+6$, and is a consequence of Corollary 2.6 and Theorem 3.2.

\begin{tabular}{|c|c|c|c|c|}
\hline $\begin{array}{c}\text { number of } \\
\text { parts of } \lambda\end{array}$ & $\begin{array}{c}\text { minimum number } \\
\text { of vertices }\end{array}$ & $\begin{array}{c}\text { minimum size } \\
\text { of each part }\end{array}$ & $\begin{array}{c}\text { lower bound } \\
\text { for } s c\left(T_{\lambda}\right)\end{array}$ & $\begin{array}{c}\text { upper bound } \\
\text { for } s c\left(T_{\lambda}\right)\end{array}$ \\
\hline 1 & 10 & 7 & $n+3$ & $n+3$ \\
2 & 19 & 8 & $n+4$ & $n+5$ \\
3 & 30 & 9 & $n+5$ & $n+7$ \\
4 & 43 & 10 & $n+6$ & $n+9$ \\
$k$ & $k^{2}+6 k+3$ & $k+6$ & $n+k+2$ & $n+2 k+1$ \\
\hline
\end{tabular}




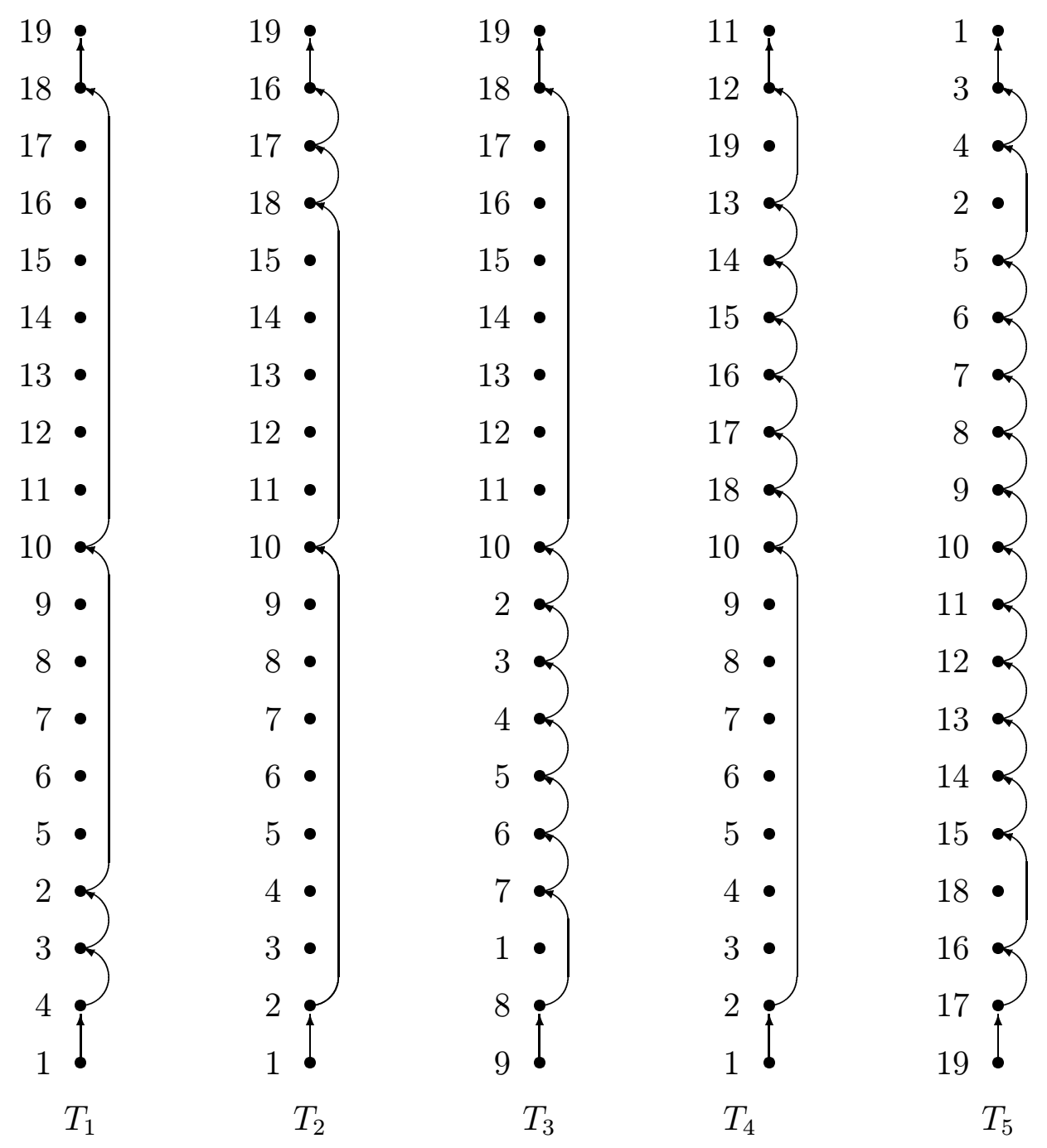

Figure 4

\section{References}

[AR] N. Alon and M. Ruszinkó, Short certificates for tournaments, The Electronic Journal of Combinatorics, (1997), 4:\# R12. 
THE ELECTRONiC JoURnAL OF COMBINATORICS 5 (1998), \#R24

[BL] R. Brualdi and Q. Li, Upsets in round robin tournaments, J. Combinatorial Theory B, (1983), 35:62-77.

[CL] G. Chartrand and L. Lesniak, Graphs and Digraphs, Third edition, Chapman and Hall, London, 1996.

[FKT] P. Fishburn, J. H. Kim, and P. Tetali, Tournament Certificates, Technical Memorandum, AT\& T Bell Laboratories, (1994), DIMACS Technical Report No. 94-05.

[KTF $] \quad$ J. H. Kim, P. Tetali, and P. Fishburn, Score certificates for tournaments, J. Graph Theory, (1997), 24:117-138.

[M] J. W. Moon, Topics on Tournaments, Holt, Rinehart, and Winston, Inc., New York, 1968.

[RB] K. B. Reid and L. W. Beineke, Selected Topics in Graph Theory (L. W. Beineke and R. J. Wilson, Eds.), Chap. 7, 385-415, Academic Press, New York, 1978.

[R] A. Rubinstein, Why are certain properties of binary relations relatively more common in natural language?, Econometrica, (1996), 64:343-355. 